\title{
Preparation of ITO Nanoparticles by Liquid Phase Coprecipitation Method
}

\author{
Zhanlai Ding, ${ }^{1}$ Cunran An, ${ }^{2}$ Qiang Li, ${ }^{1}$ Zhezhe Hou, ${ }^{1}$ Jianqiang Wang, ${ }^{1}$ Haibo Qi, \\ and Fangjuan Qi ${ }^{1}$ \\ ${ }^{1}$ School of Material Science and Engineering, Shijiazhuang Railway Institute, Shijiazhuang 050043, China \\ ${ }^{2}$ College of Science, Hebei University of Science and Technology, Shijiazhuang 050054, China \\ Correspondence should be addressed to Zhanlai Ding, ding5199@yahoo.com.cn
}

Received 16 November 2009; Revised 24 January 2010; Accepted 26 January 2010

Academic Editor: Yanqiu Zhu

Copyright ( 2010 Zhanlai Ding et al. This is an open access article distributed under the Creative Commons Attribution License, which permits unrestricted use, distribution, and reproduction in any medium, provided the original work is properly cited.

The nanoscale indium tin oxide (ITO) particles are synthesied by liquid phase coprecipitation method under given conditions with solution of indium chloride, tin chloride, and ammonia. The absolute ethyl alcohol or deionized water was used as solvent and the dodecylamine or hexadecylamine surfactant was used as a dispersant in the reaction system. The sample powder was characterized by X-ray diffraction (XRD), transmission electron microscopy (TEM), and high-resolution electron microscopy (HRTEM). Based on the transmission electron micrograph, the influences of the two different solvents and the two different dispersants on the nanoparticle size and dispersion were studied, respectively. The results showed that the ITO particles are finely crystallized bodycentered cubic structure. The particle size has distributed in $30 \mathrm{~nm}$ to $90 \mathrm{~nm}$.

\section{Introduction}

Tin-doped indium oxide (Indium tin oxide, ITO), a kind of n-type semiconductor material, has a wide forbidden band $(3.3 \mathrm{eV}$ to $4.3 \mathrm{eV})$. Indium tin oxide thin film has high transparency in the visible light region and lower electric resistivity $[1,2]$. It has been used as electrodes in manufacturing of solar cells, flat panel displays, and gas sensors. The tradition deposition techniques of ITO film are DC sputtering, RF sputtering, or electron beam evaporation. It is the first step to fabricate indium and tin alloy target or ITO ceramic target. Afterwards the target is sputtered to glass substrate by the controlled electron beam. These techniques need costly equipments, and the utilization rate of the target materials is as low as $20 \%$ [3]. Because indium is a rare metal, it is necessary to explore a new route to deposit ITO thin film with high-Indium utilization rate. The synthesis nanoparticles of metal oxide from aqueous solutions and deposition thin films at low temperatures is an important way for preparation of transparent conductive film [4]. Dip-coating or spray deposition of light transparent, good conductive, and low-membrane resistant ITO film has been studied by the researchers [5-7]. The fabrication of ITO nanoparticle is important in emulsion (so-called "ink") preparation for spray deposition or dip-coating ITO film. The ITO thin film's quality is related to the size and morphology of the nanoparticles. With the development of nanometer material research, several kinds of preparation methods for nanosized ITO emerged. The current methods for nanometer indium tin oxide preparation mainly include solid-phase method, liquid-phase method, and gas-phase method [8-10].

The liquid-phase method, with the advantages of simple operation and controllable granularity, can realize the atomic scale level of mixing. The doping of components achieves easily, and the nanoscale powder material has high-surface activity. The liquid-phase methods include liquid phase precipitation, hydrothermal (high temperature hydrolysis), Sol-gel (colloidal chemistry), radiation chemical synthesis, and so forth $[11,12]$. In this research, the ITO nanoparticles, which will be used in spray coating of ITO thin film, are prepared by liquid-phase coprecipitation with indium chloride and tin chloride as main raw material. The ITO nanoparticles were characterized by means of transmission electron microscopy (TEM), and X-ray diffraction (XRD). 


\section{Experiment Method}

2.1. Experiment Process. The synthesis process of ITO nanoparticle by liquid phase co-precipitation is as follows. A certain quality of indium chloride $\left(\mathrm{InCl}_{3} \cdot 4 \mathrm{H}_{2} \mathrm{O} 99 \%\right.$, Aldrich) and tin chloride $\left(\mathrm{SnCl}_{4} \cdot 5 \mathrm{H}_{2} \mathrm{O} 99 \%\right.$, Aldrich) was dissolved in pure deionized water or ethanol, keeping the ratio of $\mathrm{In}_{2} \mathrm{O}_{3}: \mathrm{SnO}_{2}=9: 1$. The concentrations of $\mathrm{InCl}_{3}$ in solution are $0.1,0.2$, and $0.3 \mathrm{~mol} / \mathrm{L}$, respectively. Certain concentrations $(1.25 \%, 2.50 \%$, and $5.00 \%$, resp.) of ammonia solutions were made by mixing certain amount of ammonia $\left(\mathrm{NH}_{3} \cdot \mathrm{H}_{2} \mathrm{O}, 25 \%\right)$ with pure water. The prepared $\mathrm{InCl}_{3}$ solution was transferred into fixed three-neck flask, keeping in $303 \mathrm{~K}$ to $363 \mathrm{~K}$ temperature under electromagnetic agitation. The ammonia solution was added to the flask, controlling the stirring speed and testing the $\mathrm{pH}$ value till the required $\mathrm{pH}$ value. And a certain amount of dodecylamine or hexadecylamine (DDA or HDA, Fluka) was added as dispersant. The precipitate precursor of ITO was aged a certain time and washed with deionized water and absolute alcohol for three times, respectively. After washing, the precipitates were dried for one hour at $353 \mathrm{~K}$. The dried samples were calcinated for $1,1.5$, and 2 hours at $773 \mathrm{~K}$, $873 \mathrm{~K}$, and $973 \mathrm{~K}$, respectively to get the indium tin oxide nanopowder by thermal decomposing.

2.2. Characterizing Methods. The morphology and structure of the prepared nanoparticles were characterized by means of a transmission electron microscopy (TEM) and X-ray diffraction (XRD). The microstructure of the ITO samples were analysed on a Tecnai G2 20 S-TWIN model TEM with $200 \mathrm{kV}$ acceleration voltage. For TEM analysis, the sample was dispersed in a few milliliters of tetrahydrofuran in an ultrasonic bath, and a drop of this dispersion was placed on a copper grid coated with a carbon film. The XRD measurements of nanopowder were performed on a D8 ADVANCE diffractometer with $\mathrm{Cu} \mathrm{K}_{\alpha}$ radiation $(\lambda=$ $1.5418 \AA)$.

\section{Results and Discussion}

3.1. X-Ray Diffraction of the Nanoparticles. The precursor precipitate and the calcinated samples were examined by an X-ray diffractometer (D8 ADVANCE type), respectively to analyze phase and structure. The XRD patterns are shown in Figure 1. The XRD pattern of precursor precipitate prepared in ethyl alcohol solvent is shown in Figure 1(a). A strong and sharp peak can be seen in Figure 1(a), while the other diffraction peaks are more weaker. According to JCPDS calibration (PDF\#76-1463), the precursor precipitate is $\operatorname{In}(\mathrm{OH})_{3}$ with body-centred cubic crystalline structure. The diffraction peaks are more complicated than the PDF761463 standard. The reason is that maybe there is a certain amount of $\mathrm{Sn}(\mathrm{OH})_{4}$ in the precursor. The XRD pattern in Figure $1(\mathrm{~b})$ is the result of calcinating ( $973 \mathrm{~K}$ for $1 \mathrm{~h}$ ) sample, which its precursor precipitate was prepared in pure water solvent. The XRD patterns of samples at different calcinating temperature $(773 \mathrm{~K}, 873 \mathrm{~K}$, and $973 \mathrm{~K})$ are shown in Figure 1(c), which their precursors were precipitated in ethyl alcohol solvent. According to the JCPDS (PDF\#060416), the samples in Figures 1(b) and 1 (c) are $\operatorname{In}_{2} \mathrm{O}_{3}$ powder with body-centred cubic crystalline structure. Kim, and so forth, synthesized ITO particle that had hexagonal structure and cubic structure. As the $\mathrm{pH}$ value of coprecipitated solution was increased, the amount of hexagonal crystal was decreased. ITO particle with mostly cubic structure was produced at higher than $\mathrm{pH} 10$ [12]. The ITO particles that we prepared by co-precipitate method only have cubic structure. After $793 \mathrm{~K}$, calcinating the diffraction peaks of the samples are more stronger and sharper, indicating that the $\mathrm{In}_{2} \mathrm{O}_{3}$ crystalline structure is more perfect. The crystallized characteristic is gradually becoming obvious with the calcinating temperature increasing from $773 \mathrm{~K}$ to $973 \mathrm{~K}$ by Figure $1(\mathrm{c})$. By comparison, it can be seen that the calcinating temperature plays a more important role than the calcinating time in improving crystal structure. As long as there is enough calcinating temperature, the perfect $\mathrm{In}_{2} \mathrm{O}_{3}$ nanocrystal structure can be obtained in a relatively short period of time. There are no peeks that represent the tin oxide, and the $\mathrm{In}_{2} \mathrm{O}_{3}$ lattice parameter does not change obviously, indicating that the tin atoms arrange into the $\mathrm{In}_{2} \mathrm{O}_{3}$ crystal lattice.

3.2. The TEM Analysis of ITO Nanoparticles. The particle size and morphology of the ITO samples were analyzed by a transmission electron microscopy (TEM, model Tecnai $\mathrm{G}^{2} 20$ S-TWIN). The TEM photographs of the samples are illustrated in Figure 2. The TEM photographs of the samples prepared in ethanol solvent and calcinated at $773 \mathrm{~K}$ and $973 \mathrm{~K}$, respectively are shown in Figures 2(a) and 2(b). The TEM photograph morphology in Figure 2(c) stands for the sample precipitated in deionized water solvent and calcinated at $873 \mathrm{~K}$. Comparing Figure 2(a) with Figure 2(b), it can be seen that at lower calcinating temperature $(773 \mathrm{~K})$, the particles of samples are finer, but they are more seriously agglomerated. When the thermal decomposition temperature is higher ( $973 \mathrm{~K})$, the crystal size increases obviously and the agglomeration degree is reduced. The particles appear hexagon or short rod shapes.

The particle size of indium oxide samples after $773 \mathrm{~K}^{\circ} \mathrm{C}$ calcination is 30 to $50 \mathrm{~nm}$, and the average particle size is $36.2 \mathrm{~nm}$, basing on the statistics from a group of images. It is 50 to $90 \mathrm{~nm}$ after $973 \mathrm{~K}$ calcinations, and the average particle size is $78.3 \mathrm{~nm}$. Comparing Figure 2(b) with Figure 2(c), it can be seen that although its thermo-decomposing temperature in Figure 2(b) is higher than Figure 2(c), its particle size has not grown up significantly, and that the nanoparticles dispersion has no obvious difference. Preparing in ethanol solvent does not improve the $\operatorname{In}_{2} \mathrm{O}_{3}$ nanoparticle dispersion degree. The high resolution electron microscopy (HRTEM) image of sample (b) is presented in Figure 2(d). The selected area electron diffraction (SAED) pattern is also in the same figure. The ITO nanoparticle is body-center-cubic structure and it crystallized well. 


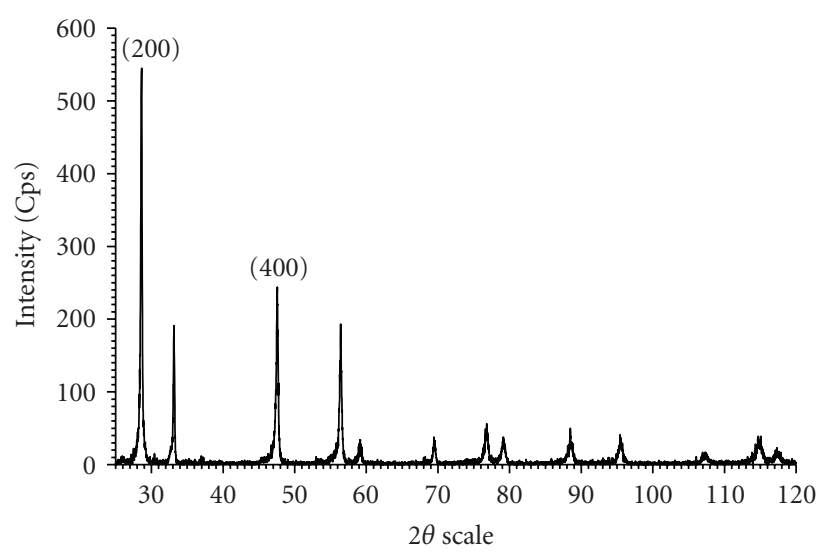

(a)

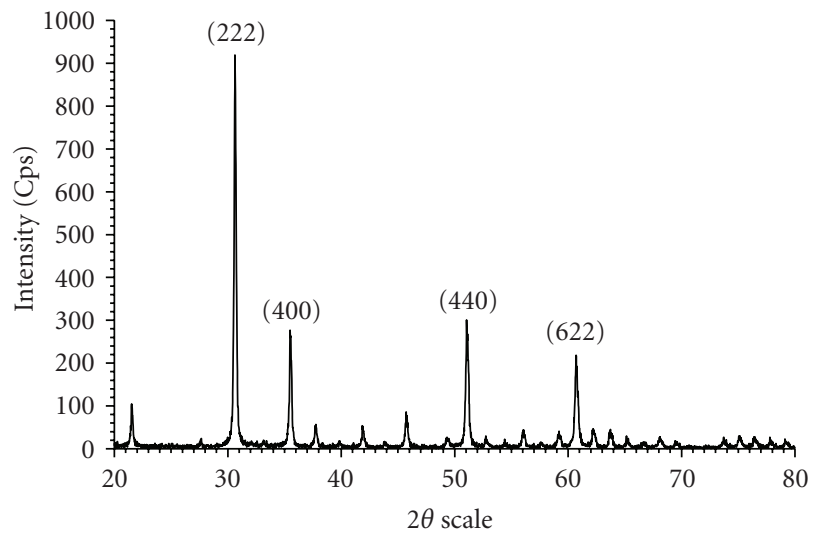

(b)

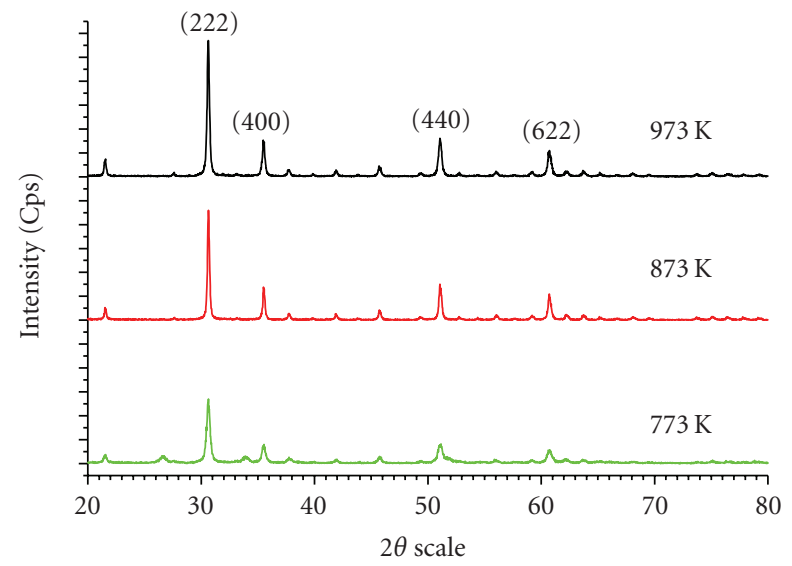

(c)

FIgURE 1: The X-ray diffraction patterns of samples. (a) The precursor precipitate, (b) the ITO nanoparticles prepared in deionized water solvent, (c) the ITO nanoparticles prepared in ethyl alcohol solvent and then decomposed at different temperatures.

3.3. The Particle Size Calculated by Scherrer Equation. The prepared ITO particles can be calculated by Scherrer equation, based on the XRD pattern. The Scherrer equation is as follow:

$$
D_{\mathrm{hkl}}=\frac{K \lambda}{\beta_{\mathrm{hkl}} \cos \theta_{\mathrm{hkl}}},
$$

where $D_{\text {hkl }}$ represents particle size (unit nm) bing perpendicular to (hkl) crystal plane. $\beta_{\mathrm{hkl}}$ is broadening (unit Rad) of the diffract peak by the grain refinement. $\theta_{\mathrm{hkl}}$ is diffraction angle of (hkl) crystal plane. $\lambda$ is the wave length of the X-ray, where it is $1.5406 \AA$. $K$ is a constant; its numerical value is related to defines of the $\beta_{\mathrm{hkl}}$. $K$ equals to 0.89 when $\beta_{\mathrm{hkl}}$ takes the half of the broadening of the diffract peak.

The particle size was calculated corresponding to the (222), (440), and (400) diffract peaks for every diffract pattern of calcinating samples. The average value of each sample is listed in Table 1.

Based on the calculated results, the particle size grows up gradually with the increase of the calcination temperature. The average particle size becomes $63 \mathrm{~nm}$ of $973 \mathrm{~K}$ calcinating from $22 \mathrm{~nm}$ of the $773 \mathrm{~K}$ calcinating. The calculated results are compared with the statistics of transmission electron
TABLE 1: The calculated results of ITO particle size by Scherrer equation.

\begin{tabular}{lccc}
\hline $\begin{array}{l}\text { Samples at different calcinating } \\
\text { temperature }(\mathrm{k})\end{array}$ & 773 & 873 & 973 \\
Average particle size value $(\mathrm{nm})$ & 22 & 44 & 63 \\
\hline
\end{tabular}

microscope. The particle size of indium tin oxide had been statistically analyzed by TEM images as in Figures 2(a) and 2(b), which the calcinating temperatures were $773 \mathrm{~K}$ and $973 \mathrm{~K}$, respectively. The particle size of indium oxide samples after $773 \mathrm{~K}$ calcination is 30 to $50 \mathrm{~nm}$, and the average particle size is $36.2 \mathrm{~nm}$. It is 50 to $100 \mathrm{~nm}$ after $973 \mathrm{~K}$ calcinations, and the average particle size is $78.3 \mathrm{~nm}$. The particle size from the TEM photograph results is larger than the calculated results by the Scherrer equation. This is mainly due to the various factors influence on the accuracy of particle size $D_{\mathrm{hkl}}$ computed by Scherrer formula, making the calculated value is less than the TEM measurements. First of all, if there is lattice imperfection in the crystal, the broadening of diffraction peak is caused not only by the grain size refinement but also by the lattice imperfection. Second, the X-ray diffraction peak is sharp. It is difficult to 


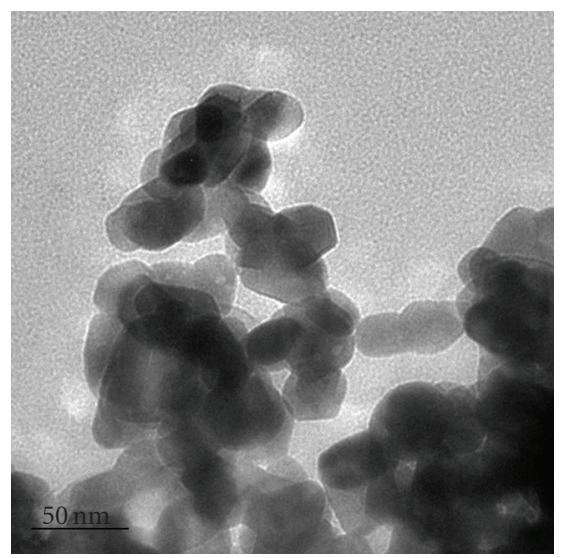

(a)

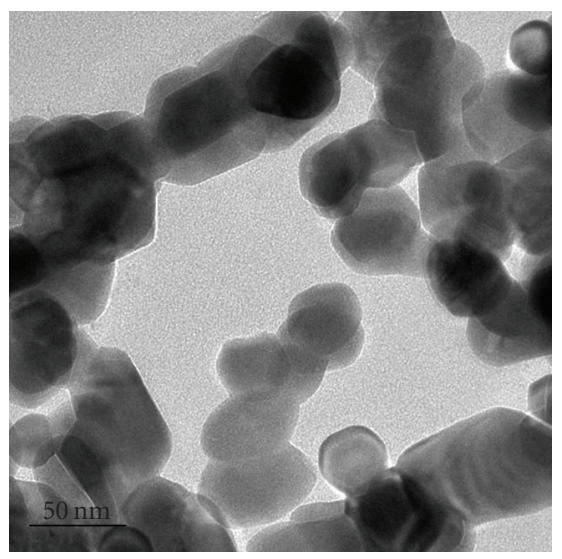

(c)

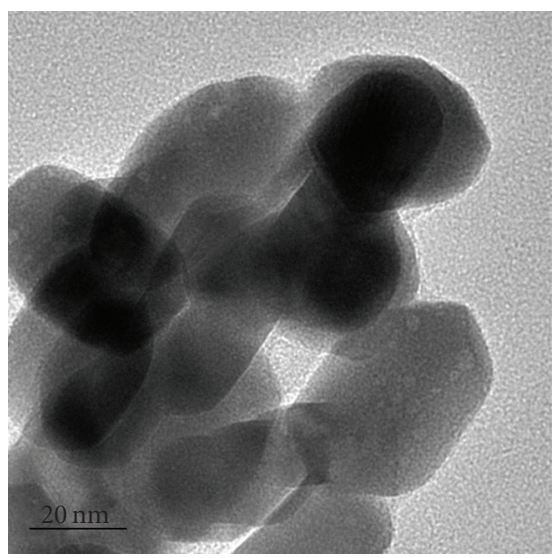

(b)

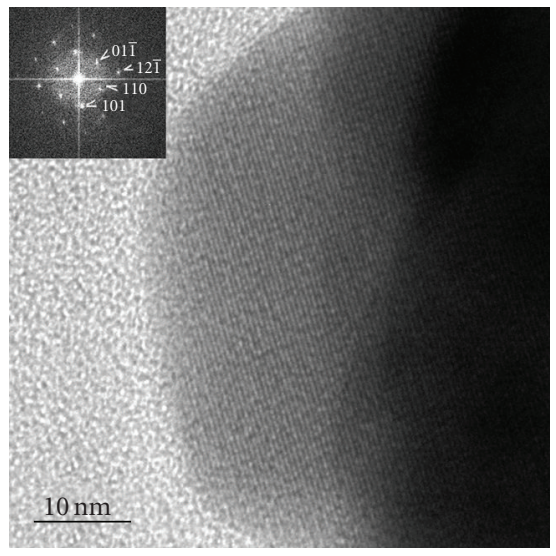

(d)

FIgURE 2: The TEM and HRTEM images of ITO nanoparticles synthesized in different solvents. (a) The absolute ethyl alcohol used as solvent and the precipitate decomposed at $737 \mathrm{~K}$, (b) the absolute ethyl alcohol used as solvent and the precipitate decomposed at $937 \mathrm{~K}$, (c) the deionized water used as solvent and the precipitate decomposed at $837 \mathrm{~K}$, (d) the HRTEM photograph and the selective electron diffraction pattern of sample (b).

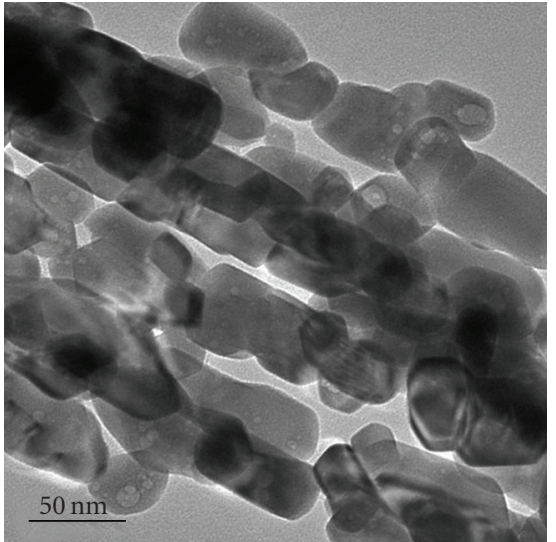

(a)

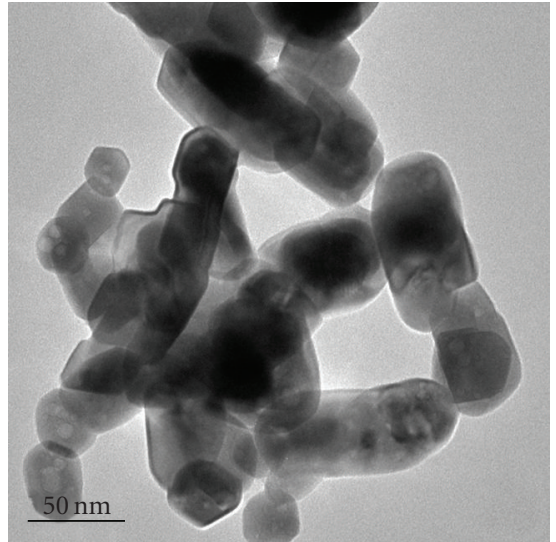

(b)

FIgURE 3: The TEM images of ITO nanoparticles with different dispersants. (a) The dodecylamine surfactant as dispersant, (b) the hexadecylamine surfactant as dispersant. 
measure the width value accurately, enlarging the calculation deviation.

3.4. Dispersant Effect on the Morphology and Dispersion of the ITO Nanoparticles. Two dispersants were used in the experiments, namely, dodecylamine and hexadecylamine. The dispersion performance of ITO nanoparticle was compared through the contrast experiment. The ITO nanoparticle TEM images, which prepared under the same conditions except using two kinds of dispersant, respectively, are shown in Figure 3. The ITO nanoparticle TEM photo with dodecylamine surfactant as dispersant is in Figure 3(a). While the ITO nanoparticle TEM photo with hexadecylamine surfactant as dispersant is in Figure 3(b). It can be observed from Figure 3 that ITO nanoparticles synthesized with dodecylamine are not dispersed well, and some particles are arranged into the long axis orderly. At the same time, the ITO nanoparticles synthesized with hexadecylamine are dispersed better than with dodecylamine. The morphology of ITO nanoparticles either with dodecylamine or with hexadecylamine as dispersant appears irregular shape. Some of the particles appear hexagon or short rod shapes.

\section{Conclusion}

The nanoscale indium tin oxide (ITO) particles are successfully synthesized by liquid phase co-precipitation method under given conditions with reactants of indium chloride, tin chloride, and ammonia. The absolute ethyl alcohol or deionized water was used as solvent, and the dodecylamine or hexadecylamine surfactant was used as a dispersant in the reaction system. The ITO particles are finely crystallized body centered cubic structure. The particle size has preferably dispersivity and distributes in $30 \mathrm{~nm}$ to $90 \mathrm{~nm}$.

The dispersivity of ITO nanoparticle does not have significant difference prepared in absolute ethyl alcohol or deionized water solvent with hexadecylamine surfactant as dispersant in the reaction system. At certain conditions, the dodecylamine surfactant can organise the indium oxide nanoparticles in lines, but that self-assembly phenomena needs further study.

\section{Acknowledgments}

The author is thankful for the financial support of Natural Science Foundation of Hebei Province (no: E2008000429). The author also thanks Dr. Zhang of Nano-science Research Center for the TEM analysis and the discussions on the results.

\section{References}

[1] G. Cheng, E. Stern, S. Guthrie, et al., "Indium oxide nanostructures," Applied Physics A, vol. 85, no. 3, pp. 233-240, 2006.

[2] F. O. Adurodija, L. Semple, and R. Brüning, "Crystallization process and electro-optical properties of $\mathrm{In}_{2} \mathrm{O}_{3}$ and ITO thin films," Journal of Materials Science, vol. 41, no. 21, pp. 70967102, 2006.
[3] S. L. Wang and D. L. Xia, "Fabrication techniques and development of ITO film," GLASS \& ENAMEL, vol. 32, no. 5, pp. 51-54, 2004.

[4] T. P. Niesen and M. R. De Guire, "Review: deposition of ceramic thin films at low temperatures from aqueous solutions," Journal of Electroceramics, vol. 6, no. 3, pp. 169207, 2001.

[5] U. Betz, M. Kharrazi Olsson, J. Marthy, M. F. Escolá, and F. Atamny, "Thin films engineering of indium tin oxide: large area flat panel displays application," Surface and Coatings Technology, vol. 200, no. 20-21, pp. 5751-5759, 2006.

[6] T. Ogi, F. Iskandar, Y. Itoh, and K. Okuyama, "Characterization of dip-coated ITO films derived from nanoparticles synthesized by low-pressure spray pyrolysis," Journal of Nanoparticle Research, vol. 8, no. 3-4, pp. 343-350, 2006.

[7] W. Chang, S. Lee, C. Yang, and T. Lin, "Opto-electronic properties of chromium doped indium-tin-oxide films deposited at room temperature," Materials Science and Engineering B, vol. 153, no. 1-3, pp. 57-61, 2008.

[8] Y. Zhang, H. Ago, J. Liu, et al., "The synthesis of $\mathrm{In}, \mathrm{In}_{2} \mathrm{O}_{3}$ nanowires and $\operatorname{In}_{2} \mathrm{O}_{3}$ nanoparticles with shape-controlled," Journal of Crystal Growth, vol. 264, no. 1-3, pp. 363-368, 2004.

[9] K. Soulantica, L. Erades, M. Sauvan, F. Senocq, A. Maisonnat, and B. Chaudret, "Synthesis of indium and indium oxide nanoparticles from indium cyclopentadienyl precursor and their application for gas sensing," Advanced Functional Materials, vol. 13, no. 7, pp. 553-557, 2003.

[10] H. S. Kim, P. D. Byrne, A. Facchetti, and T. J. Marks, "High performance solution-processed indium oxide thinfilm transistors," Journal of the American Chemical Society, vol. 130, no. 38, pp. 12580-12581, 2008.

[11] S. Deki, S. Iizuka, M. Mizuhata, and A. Kajinami, "Fabrication of nano-structured materials from aqueous solution by liquid phase deposition," Journal of Electroanalytical Chemistry, vol. 584, no. 1, pp. 38-43, 2005.

[12] A. Nakata, M. Mizuhata, and S. Deki, "Novel fabrication of highly crystallized nanoparticles in the confined system by the liquid phase deposition (LPD) method," Electrochimica Acta, vol. 53, no. 1, pp. 179-185, 2007. 

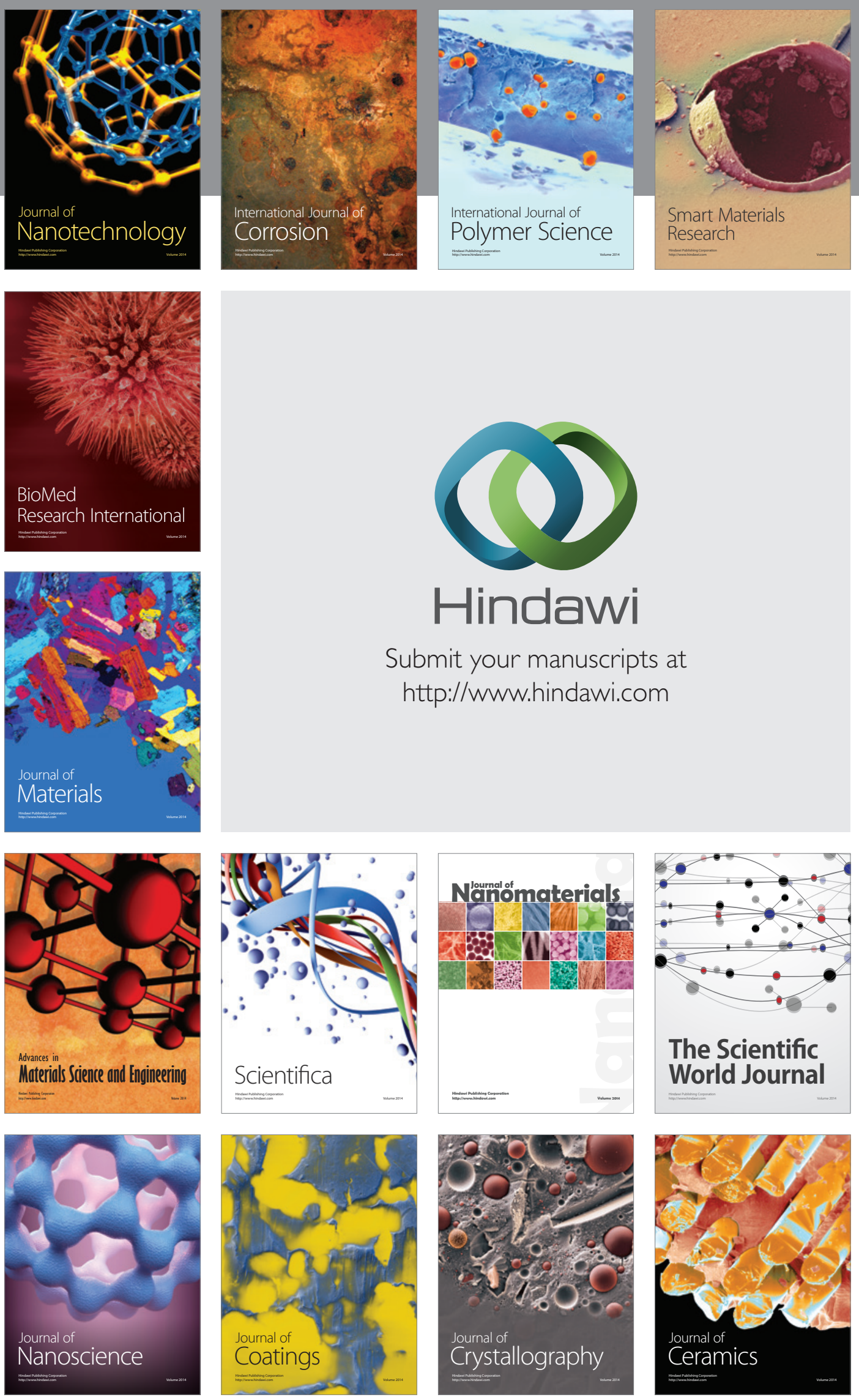

The Scientific World Journal

Submit your manuscripts at

http://www.hindawi.com

\section{World Journal}

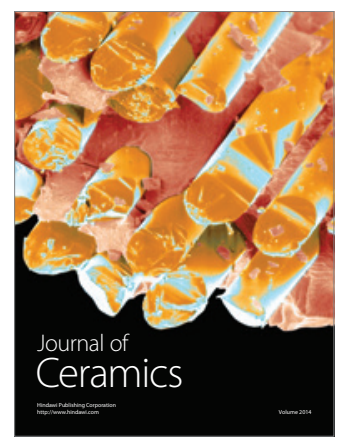

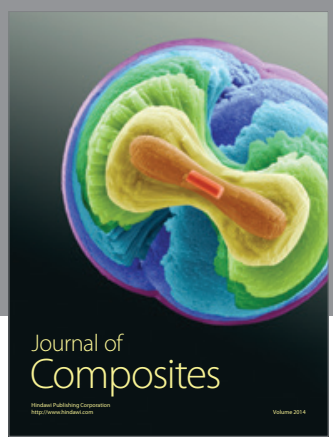
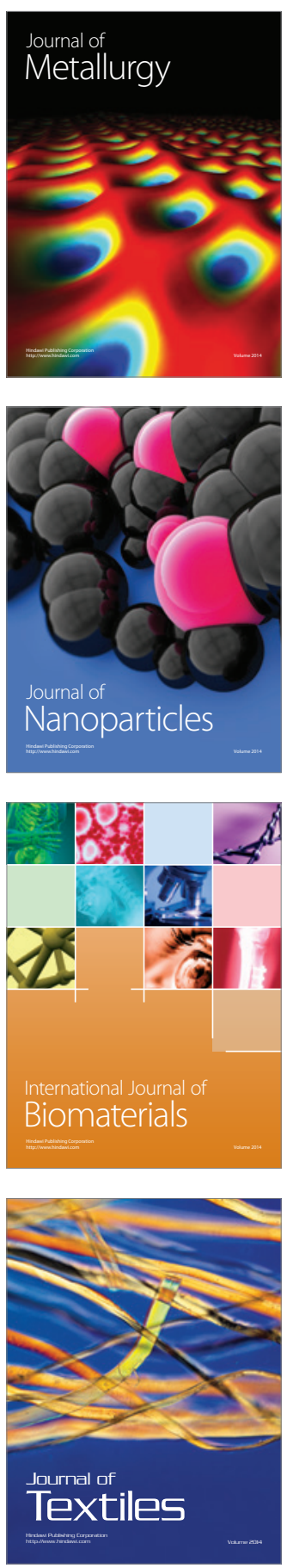